\title{
Cytokeratin 5/6 fingerprinting in HER2-positive tumors identifies a poor prognosis and trastuzumab-resistant Basal- HER2 subtype of breast cancer
}

\author{
Begoña Martin-Castillo,2, Eugeni Lopez-Bonet ${ }^{2,3}$, Maria Buxó ${ }^{2,4,5}$, Joan Dorca ${ }^{6}$, \\ Francesc Tuca-Rodríguez ${ }^{7}$, Miguel Alonso Ruano7, Ramon Colomer,9, Javier \\ A. Menendez 2,10 \\ ${ }^{1}$ Unit of Clinical Research, Catalan Institute of Oncology, Girona, Catalonia, Spain \\ ${ }^{2}$ Girona Biomedical Research Institute (IDIBGI), Molecular Oncology Group, Girona, Catalonia, Spain \\ $3^{3}$ Department of Anatomical Pathology, Dr. Josep Trueta Hospital of Girona, Girona, Catalonia, Spain \\ ${ }^{4}$ Epidemiology Unit and Cancer Registry of Girona (UERCG), Catalan Cancer Plan, Catalan Health Government, Girona, \\ Catalonia, Spain \\ ${ }^{5}$ Department of Nursing, Universitat de Girona (UdG), Girona, Catalonia, Spain \\ ${ }^{6}$ Medical Oncology Department, Catalan Institute of Oncology, Girona, Catalonia, Spain \\ ${ }^{7}$ Department of Gynecology, Dr. Josep Trueta Hospital of Girona, Girona, Catalonia, Spain \\ ${ }^{8}$ Breast Cancer Clinical Research Unit, CNIO-Spanish National Cancer Research Center, Madrid, Spain \\ ${ }^{9}$ Medical Oncology Department, Hospital La Princesa, Madrid, Spain \\ ${ }^{10}$ Translational Research Laboratory, Catalan Institute of Oncology (ICO), Girona, Catalonia, Spain \\ Correspondence to: \\ Javier A. Menendez, e-mail: jmenendez@iconcologia.net, jmenendez@idibgi.org \\ Keywords: Breast cancer, HER2, basal-like, trastuzumab, cytokeratins \\ Received: December 22, $2014 \quad$ Accepted: January 08, $2015 \quad$ Published: January 29, 2015
}

\section{ABSTRACT}

There is an urgent need to refine the prognostic taxonomy of HER2+ breast carcinomas and develop easy-to-use, clinic-based prediction algorithms to distinguish between good- and poor- responders to trastuzumab-based therapy. Building on earlier studies suggesting that HER2+ tumors enriched with molecular and morphoimmunohistochemical features classically ascribed to basal-like tumors are highly aggressive and refractory to trastuzumab, we investigated the prognostic and predictive value of the basal-HER2+ phenotype in HER2-overexpressing tumors. Our retrospective cohort study of a consecutive series of 152 HER2+ primary invasive ductal breast carcinomas first confirmed the existence of a distinct subgroup co-expressing HER2 protein and basal cytokeratin markers CK5/6, the so-called basalHER2+ phenotype. Basal-HER2+ phenotype ( $\geq 10 \%$ of cells showing positive CK5/6 staining), but not estrogen receptor status, was significantly associated with inferior overall survival by univariate analysis and predicted worsened disease free survival after accounting for strong prognostic variables such as tumor size at diagnosis in stepwise multivariate analysis. In the sub-cohort of HER2+ patients treated with trastuzumab-based adjuvant/neoadjuvant therapy, basal-HER2+ phenotype was found to be the sole independent prognostic marker for a significantly inferior time to treatment failure in multivariate analysis. A CK5/6-based immunohistochemical fingerprint may provide a simple, rapid, and accurate method for re-classifying women diagnosed with HER2+ breast cancer in a manner that can improve prognosis and therapeutic planning in patients with clinically aggressive basal-HER2+ tumors who are not likely to benefit from trastuzumab-based therapy. 
In women with early-stage breast cancer, adjuvant use of the anti-HER2 monoclonal antibody trastuzumab reduces recurrence risk when added to standard chemotherapy [1, 2]. However, not all cases of HER2+ breast tumors derive similar benefit from trastuzumab because a significant number of patients develop disease recurrence. At present, clinicians rely on established markers of HER2 expression for selecting patients for adjuvant trastuzumab or neoadjuvant pertuzumab-trastuzumab therapy i.e., immunohistochemical expression at the $3+$ level or FISH ratio of 2 or greater [3]. Biomarkers to identify those patients who are not likely to benefit from trastuzumab would be clinically useful, allowing patients to pursue other therapeutic options. A predictive biomarker to classify a subgroup of patients with HER2+ tumors that are particularly resistant to trastuzumab could be especially important in the adjuvant setting, where the effectiveness of a given therapy in an individual patient cannot be assessed. Unfortunately, the identification of a robust clinical or molecular predictor of adjuvant trastuzumab benefit, including HER2 itself, has proven challenging [4-8].

Earlier work by Harris et al. [9], demonstrated that a particular HER2+ tumor phenotype overexpressing genes associated with the basal-like phenotype, including higher expression of basal cytokeratins (CKs), was more frequent in the non-responding group of patients receiving pre-operative trastuzumab than in the responding group. Building on these pioneering findings and considering that trastuzumab sensitivity is notably restricted to luminal-HER2+ breast cancer cell lines, whereas all basalHER2 + cell lines exhibit inherent primary resistance to trastuzumab [10], we recently proposed that a basal CK surrogate definition of HER2+ breast carcinomas might define subgroups of patients likely to display resistance to trastuzumab-based therapy. Although published data on HER2 + and basal phenotype are limited, the longstanding assumption that HER2+ and basal-like breast cancers are mutually exclusive entities is open to dispute. While the first molecular portraits of breast tumors using DNA microarrays suggested that breast carcinomas with a basal phenotype are HER2 non-amplified [11], subsequent refined analyses tended to include HER2-amplified tumors branching close or included in a bona fide basallike subclass [12-15]. Beyond microarray-based gene profiling studies, the so-called basal-HER2+ subtype has been also identified by immunohistochemical biomarker profiles. When Laakso et al. segregated basal-like breast cancers based on immunohistochemical expression of basal CKs, those tumors with low basal CK expression were likely to have HER2 overexpression [16]. Similarly, Liu et al. described a small group of hormone receptornegative tumors simultaneously expressing HER2 and basal markers [17]. Both studies found that patients with the basal-HER2 + subtype had a significantly worse prognosis than those with basal-like and HER2+ tumors. Bhargava et al. [18] and our own group [19] observed that many morphological and immunohistochemical features classically ascribed to basal-like tumors, including large geographic necrosis and lymphoid infiltrate, are commonly seen in basal-HER2+ tumors. Bagaria et al. [20] have recently confirmed that when luminalHER2+ (ER-positive and basal CK-negative), HER2+ (ER-negative and basal CK-negative) and basal-HER2+ (ER-negative and basal CK-positive) were correlated with clinicopathological features and overall survival, the basal-HER2+ subtype was associated with the worst prognosis.

Collectively, the results from these studies strongly support the notion that the basal-HER2+ phenotype may delineate a distinct entity of biologically-aggressive breast carcinomas; however, whether the basal-HER2+ phenotype also has clinical utility as a predictive marker of resistance to trastuzumab-based therapy remains to be clarified. Beyond confirming that the basal-HER2+ phenotype can predict worse diseasefree and overall survival, we have also evaluated whether immunohistochemical-based identification of the basal-HER2+ phenotype can predict resistance to trastuzumab-based adjuvant therapy, which may have crucial implications for patients originally identified as suitable for trastuzumab based solely on their HER2+ phenotype. We show here that a simple CK5/6-based fingerprint using a $10 \%$ positivity cutoff, allows the re-classification of HER2+ tumors in a manner that improves prognosis and therapeutic planning in a subclass of patients with clinically aggressive basal-HER2+ tumors that are not likely to benefit from trastuzumabbased therapy.

\section{RESULTS}

\section{Clinicopathological features}

Breast cancer tissue sections of 154 consecutive patients with HER2-overexpressing primary invasive ductal breast cancer were evaluated for expression of ER and CK5/6 by IHC: 89 (58\%) tumors were luminal-HER2+, $39(26 \%)$ tumors were HER2+, and $24(16 \%)$ tumors were basal-HER+ (Table 1). Representative examples of HER2+ breast carcinomas expressing basal epithelial CK markers $(\mathrm{CK} 5 / 6)$ are shown in Figure 1.

Patients with basal-HER2+ tumors were more likely to have larger tumors $(P=0.011)$ and recurrence $(P=0.007)$ than those with luminal-HER2+ and HER2+ tumors. There were no differences in age at diagnosis, tumor size, nodal status, and tumor grade between patients with luminal-HER2+ and HER2+ tumors. There were no differences in the use of adjuvant trastuzumab and chemotherapy across the three HER2+ groups. Patients with luminal-HER2+ tumors were more likely to receive hormone therapy than patients with HER2+ and basalHER2+ tumors $(P<0.001)$ (Table 1$)$. 
Table 1: Patient and tumor characteristics

\begin{tabular}{|c|c|c|c|c|c|}
\hline Characteristic & $\begin{array}{c}\text { Luminal-HER2+ } \\
n(\%)\end{array}$ & $\begin{array}{c}\text { Basal-HER2+ } \\
n(\%)\end{array}$ & $\begin{array}{l}\text { HER2+ } \\
n(\%)\end{array}$ & $\begin{array}{c}\text { Basal-HER2+ } \\
\text { vs others } \\
P \text { value*a }\end{array}$ & $\begin{array}{c}\text { Luminal-HER2+ } \\
\text { vs HER2+ } \\
P \text { value }{ }^{* a}\end{array}$ \\
\hline No. of patients & $89(58.6)$ & $24(15.8)$ & $39(25.7)$ & & \\
\hline Age, years, mean $\pm \mathrm{SD}$ & $58.2 \pm 16.4$ & $63.1 \pm 15.3$ & $56.67 \pm 14.2$ & $0.130 * 1$ & $0.591 * 1$ \\
\hline \multicolumn{6}{|l|}{ Tumor size } \\
\hline $\mathrm{T} 1$ & $34(38.2)$ & $3(12.5)$ & $13(33.3)$ & $0.011 / 0.008 * 2$ & $0.350 / 0.345^{* 2}$ \\
\hline $\mathrm{T} 2$ & $41(46.1)$ & $10(41.7)$ & $17(43.6)$ & & \\
\hline $\mathrm{T} 3+\mathrm{T} 4$ & $6(6.7)$ & $8(33.3)$ & $7(17.9)$ & & \\
\hline Inflammatory & $5(5.6)$ & $2(8.3)$ & $2(5.1)$ & & \\
\hline Unknown & $3(3.4)$ & $1(4.2)$ & $0(0.0)$ & & \\
\hline \multicolumn{6}{|l|}{ Node status } \\
\hline Negative & $42(47.2)$ & $7(29.2)$ & $19(48.7)$ & $0.177 / 0.097 * 2$ & $0.566 / 0.943 * 2$ \\
\hline Positive & $43(48.3)$ & $16(66.7)$ & $20(51.3)$ & & \\
\hline Unknown & $4(4.5)$ & $1(4.2)$ & $0(0.0)$ & & \\
\hline \multicolumn{6}{|l|}{ Tumor grade } \\
\hline 1 & $3(3.4)$ & $0(0.0)$ & $1(2.6)$ & $0.934 / 0.892 * 2$ & $0.435 / 0.478$ \\
\hline 2 & $25(28.1)$ & $5(20.8)$ & $8(20.5)$ & & \\
\hline 3 & $43(48.3)$ & $14(58.3)$ & $25(64.1)$ & & \\
\hline Unknown & $18(20.2)$ & $5(20.8)$ & $5(12.8)$ & & \\
\hline \multicolumn{6}{|l|}{ Adjuvant trastuzumab } \\
\hline No & $40(44.9)$ & $13(54.2)$ & $20(51.3)$ & $0.514 / 0.738 * 2 * b$ & $0.793 / 0.594 * 2 * b$ \\
\hline Yes & $42(47.2)$ & $11(45.8)$ & $17(43.6)$ & & \\
\hline Unknown & $7(7.9)$ & $0(0.0)$ & $0(0.0)$ & & \\
\hline \multicolumn{6}{|l|}{ Adjuvant chemotherapy } \\
\hline No & $21(23.6)$ & $4(16.7)$ & $8(20.5)$ & $0.143 / 0.394 * 2 * b$ & $0.878 / 0.647 * 2 * b$ \\
\hline Yes & $57(64.0)$ & $20(83.3)$ & $27(69.2)$ & & \\
\hline Unknown & $11(12.4)$ & $0(0.0)$ & $4(10.3)$ & & \\
\hline \multicolumn{6}{|l|}{ Adjuvant hormone therapy } \\
\hline No & $7(7.9)$ & $21(87.5)$ & $37(94.4)$ & $<0.001 /<0.001 * 2 * b$ & $<0.001 /<0.001 * 2 * b$ \\
\hline Yes & $71(79.8)$ & $3(12.5)$ & $2(5.1)$ & & \\
\hline Unknown & $11(12.4)$ & $0(0.0)$ & $0(0.0)$ & & \\
\hline \multicolumn{6}{|l|}{$\begin{array}{l}\text { First clinically relevant } \\
\text { event }\end{array}$} \\
\hline Local recurrence & $1(1.1)$ & $2(8.3 \%)$ & $1(2.6)$ & $0.007 / 0.004 * 2$ & $0.699 / 0.861 * 2$ \\
\hline Distant metastasis & $11(12.4)$ & $7(29.2)$ & $7(18.4)$ & & \\
\hline $\begin{array}{l}\text { Contralateral breast } \\
\text { cancer }\end{array}$ & $0(0.0)$ & $2(8.3)$ & $0(0.0)$ & & \\
\hline Second primary tumor & $5(5.6)$ & $1(4.2)$ & $2(5.3)$ & & \\
\hline Not occurred & $58(65.2)$ & $9(37.5)$ & $24(63.2)$ & & \\
\hline
\end{tabular}




\begin{tabular}{|c|c|c|c|c|c|}
\hline Characteristic & $\begin{array}{c}\text { Luminal-HER2+ } \\
n(\%)\end{array}$ & $\begin{array}{c}\text { Basal-HER2+ } \\
n(\%)\end{array}$ & $\begin{array}{l}\text { HER2+ } \\
n(\%)\end{array}$ & $\begin{array}{c}\text { Basal-HER2+ } \\
\text { vs others } \\
P \text { value*a }\end{array}$ & $\begin{array}{c}\text { Luminal-HER2+ } \\
\text { vs HER2+ } \\
P_{\text {value }}^{* a}\end{array}$ \\
\hline Unknown & $8(9.0)$ & $1(4.2)$ & $1(2.6)$ & & \\
\hline Death from other causes & $6(6.7)$ & $2(8.3)$ & $3(7.9)$ & & \\
\hline \multicolumn{6}{|l|}{ First metastases site } \\
\hline Not occured & $79(88.8)$ & $17(70.8)$ & 33 (84.6) & 0.084 & 0.773 \\
\hline Visceral & $3(3.4)$ & $2(8.3)$ & $1(2.6)$ & & \\
\hline No visceral & $4(4.5)$ & $2(8.3)$ & $3(7.7)$ & & \\
\hline Both & $3(3.4)$ & $3(12.5)$ & $2(5.1)$ & & \\
\hline
\end{tabular}

*1 Parametric test: Independent two-sample Student's $t$ test

*2 excludes unknown category

*a Fisher's exact test

${ }^{* b}$ Chi-square test

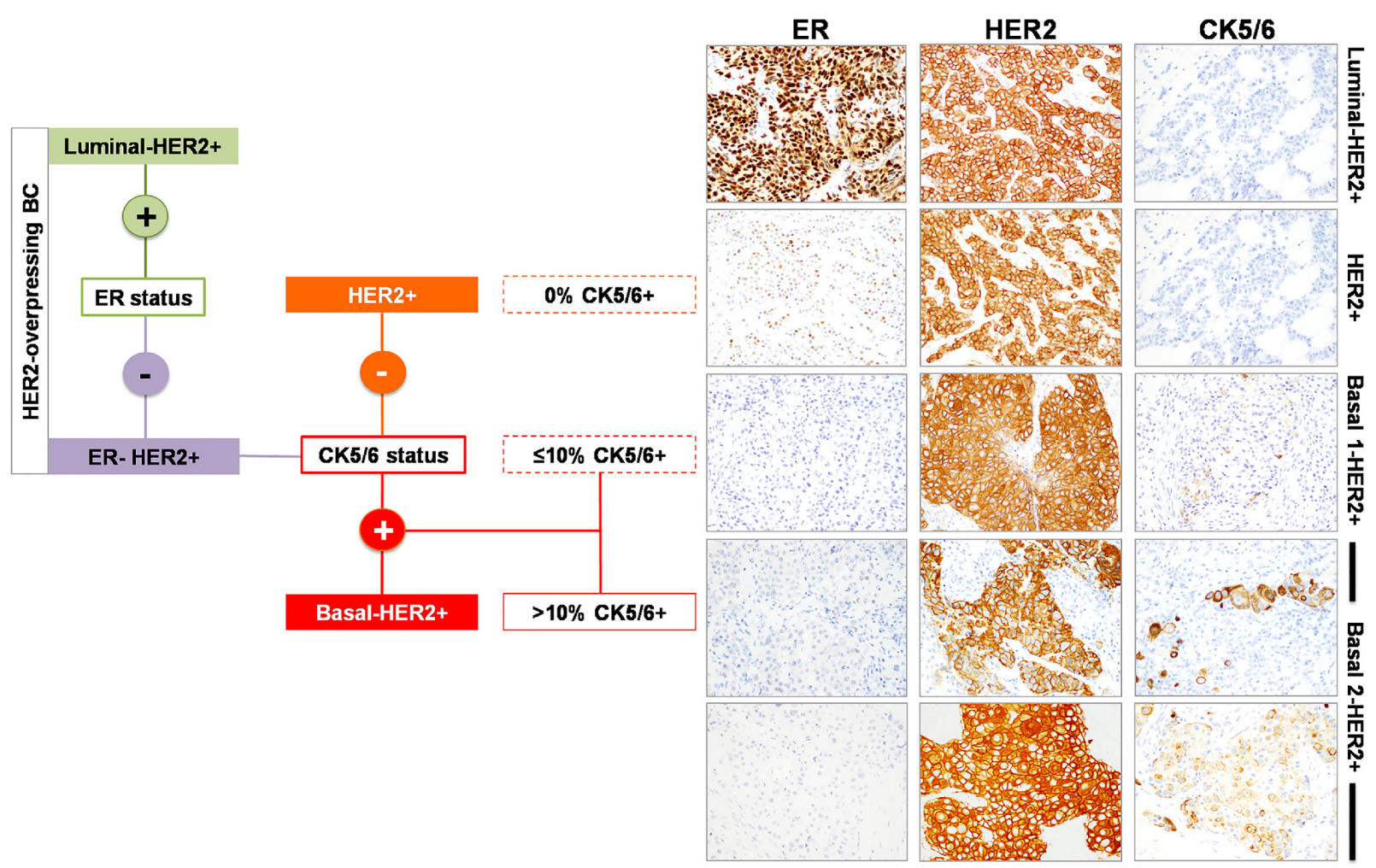

Figure 1: Immunophenotypic classification of HER2-overexpressing breast carcinomas.

To exclude equivocal reactions, clinicopathological features were reassessed after stratification of the basalHER2+ tumors $(\mathrm{CK} 5 / 6$ staining score $>0)$ to underlying CK5/6 expression pattern: basal 1-HER $2+(<10 \%$ of cells showing positive staining) and basal $2 /$ HER $2+(\geq 10 \%$ of cells showing positive staining) (Table 2 ). When a positive CK5/6 staining in $\geq 10 \%$ of the HER $2+$ breast cancer tissue sections was registered as a diagnostically relevant positive reaction [21], patients with basal 2-HER2+ tumors (but not basal 1-HER2+ tumors) were older $(P=0.034)$ and more likely to have larger tumors $(P=0.003)$ and recurrence $(P<0.001)$ than those with luminal-HER $2+$ and HER2+ tumors. When compared to HER2+ tumors, patients with basal 2-HER2 tumors (but not basal 1-HER2+ tumors) were older $(P=0.035)$ more likely to have larger tumors $(P=0.022)$ and recurrence $(P=0.012)$ (Table 2$)$. 
Table 2: Patient and tumor characteristics (stratification by CK5/6 expression status)

\begin{tabular}{|c|c|c|c|c|c|c|c|c|}
\hline Characteristic & $\begin{array}{c}\text { Luminal- } \\
\text { HER2+ } \\
n(\%)\end{array}$ & $\begin{array}{c}\text { Basal } \\
\text { 1-HER2+ } \\
n(\%)\end{array}$ & $\begin{array}{c}\text { Basal } \\
\text { 2-HER2+ } \\
n(\%)\end{array}$ & $\begin{array}{c}\text { HER2+ } \\
n(\%)\end{array}$ & $\begin{array}{c}\text { Basal } \\
\text { 1-HER2+ } \\
\text { vs others } \\
P \text { value }^{* a}\end{array}$ & $\begin{array}{c}\text { Basal2- } \\
\text { HER2+ vs } \\
\text { others } \\
P \text { value }^{*_{a}}\end{array}$ & $\begin{array}{c}\text { Basal1- } \\
\text { HER2+ vs } \\
\text { HER2+ } \\
P \text { value*a }\end{array}$ & $\begin{array}{c}\text { Basal2- } \\
\text { HER+ vs } \\
\text { HER2+ } \\
P \text { value*a }\end{array}$ \\
\hline No. of patients & $89(58.6)$ & $12(7.9)$ & $12(7.9)$ & $39(25.7)$ & & & & \\
\hline Age, years, mean \pm SD & $\begin{array}{c}58.2 \pm \\
16.4\end{array}$ & $\begin{array}{c}57.83 \pm \\
14.0\end{array}$ & $\begin{array}{l}68.33 \pm \\
15.3\end{array}$ & $\begin{array}{c}56.67 \pm \\
14.2\end{array}$ & $0.935^{* 1}$ & $0.034^{* 1}$ & $0.739 * 1$ & $0.035^{* 1}$ \\
\hline \multicolumn{9}{|l|}{ Tumor size } \\
\hline $\mathrm{T} 1$ & $34(38.2)$ & $3(25.0)$ & $0(0.0)$ & $13(33.3)$ & $\begin{array}{l}0.483 / \\
0.360 * 2\end{array}$ & $\begin{array}{l}0.003 / \\
0.002 * 2\end{array}$ & $\begin{array}{l}0.849 / \\
0.849 * 2\end{array}$ & $\begin{array}{l}0.022 / \\
0.048 * 2\end{array}$ \\
\hline $\mathrm{T} 2$ & $41(46.1)$ & $5(41.7)$ & $5(41.7)$ & $17(43.6)$ & & & & \\
\hline $\mathrm{T} 3+\mathrm{T} 4$ & $6(6.7)$ & $3(25.0)$ & $5(41.7)$ & 7 (17.9) & & & & \\
\hline Inflammatory & $5(5.6)$ & $1(8.3)$ & $1(8.3)$ & $2(5.1)$ & & & & \\
\hline Unknown & $3(3.4)$ & $0(0.0)$ & $1(8.3)$ & $0(0.0)$ & & & & \\
\hline \multicolumn{9}{|l|}{ Node status } \\
\hline Negative & $42(47.2)$ & $2(16.7)$ & $5(41.7)$ & $19(48.7)$ & $\begin{array}{l}0.092 / \\
0.036^{* 2}\end{array}$ & $\begin{array}{l}0.515 / \\
0.531 * 2\end{array}$ & $\begin{array}{l}0.091 / \\
0.091 * 2\end{array}$ & $\begin{array}{l}0.311 / \\
1.000 * 2\end{array}$ \\
\hline Positive & $43(48.3)$ & $10(83.3)$ & $6(50.0)$ & $20(51.3)$ & & & & \\
\hline Unknown & $4(4.5)$ & $0(0.0)$ & $1(8.3)$ & $0(0.0)$ & & & & \\
\hline \multicolumn{9}{|l|}{ Tumor grade } \\
\hline 1 & $3(3.4)$ & $0(0.0)$ & $0(0.0)$ & $1(2.6)$ & $\begin{array}{l}0.847 / \\
1.000 * 2\end{array}$ & $\begin{array}{l}0.656 / \\
1.000 * 2\end{array}$ & $\begin{array}{l}1.000 / \\
1.000 * 2\end{array}$ & $\begin{array}{l}0.453 / \\
1.000 * 2\end{array}$ \\
\hline 2 & $25(28.1)$ & $3(25.0)$ & $2(16.7)$ & $8(20.5)$ & & & & \\
\hline 3 & $43(48.3)$ & $8(66.7)$ & $6(50.6)$ & $25(64.1)$ & & & & \\
\hline Unknown & $18(20.2)$ & $1(8.3)$ & $4(33.3)$ & $5(12.8)$ & & & & \\
\hline \multicolumn{9}{|l|}{ Adjuvant trastuzumab } \\
\hline No & $40(44.9)$ & $4(33.3)$ & $9(75.0)$ & $20(51.3)$ & $\begin{array}{l}0.414 / \\
0.366^{* 2}\end{array}$ & $\begin{array}{c}0.207 / \\
0.104^{* 2 * b}\end{array}$ & $\begin{array}{c}0.420 / \\
0.212 * 2 * \mathrm{~b}\end{array}$ & $\begin{array}{l}0.413 / \\
0.313 * 2\end{array}$ \\
\hline Yes & $42(47.2)$ & $8(66.7)$ & $3(25.0)$ & $17(43.6)$ & & & & \\
\hline Unknown & $7(7.9)$ & $0(0.0)$ & $0(0.0)$ & $0(0.0)$ & & & & \\
\hline \multicolumn{9}{|l|}{ Adjuvant chemotherapy } \\
\hline No & $21(23.6)$ & $1(8.3)$ & $3(25.0)$ & $8(20.5)$ & $\begin{array}{l}0.271 / \\
0.291 * 2\end{array}$ & $\begin{array}{l}0.587 / \\
1.000 * 2\end{array}$ & $\begin{array}{l}0.448 / \\
0.412 * 2\end{array}$ & $\begin{array}{l}0.744 / \\
1.000 * 2\end{array}$ \\
\hline Yes & $57(64.0)$ & $11(91.7)$ & $9(75.0)$ & $27(69.2)$ & & & & \\
\hline Unknown & $11(12.4)$ & $0(0.0)$ & $0(0.0)$ & $4(10.3)$ & & & & \\
\hline \multicolumn{9}{|l|}{$\begin{array}{l}\text { Adjuvant hormone } \\
\text { therapy }\end{array}$} \\
\hline No & $7(7.9)$ & $9(75.0)$ & $12(100.0)$ & $37(94.4)$ & $\begin{array}{l}0.031 / \\
0.027 * 2\end{array}$ & $\begin{array}{c}<0.001 / \\
<0.001 * 2 * \mathrm{~b} \\
\end{array}$ & $\begin{array}{l}0.078 / \\
0.078 * 2 \\
\end{array}$ & $\begin{array}{l}1.000 / \\
1.000 * 2\end{array}$ \\
\hline Yes & $71(79.8)$ & $3(25.0)$ & $0(0.0)$ & $2(5.1)$ & & & & \\
\hline Unknown & $11(12.4)$ & $0(0.0)$ & $0(0.0)$ & $0(0.0)$ & & & & \\
\hline
\end{tabular}

(Continued) 


\begin{tabular}{|c|c|c|c|c|c|c|c|c|}
\hline Characteristic & $\begin{array}{c}\text { Luminal- } \\
\text { HER2+ } \\
n(\%)\end{array}$ & $\begin{array}{c}\text { Basal } \\
\text { 1-HER2+ } \\
n(\%)\end{array}$ & $\begin{array}{c}\text { Basal } \\
\text { 2-HER2+ } \\
n(\%)\end{array}$ & $\begin{array}{c}\text { HER2+ } \\
n(\%)\end{array}$ & $\begin{array}{c}\text { Basal } \\
\text { 1-HER2+ } \\
\text { vs others } \\
P \text { value*a }\end{array}$ & $\begin{array}{c}\text { Basal2- } \\
\text { HER2+ vs } \\
\text { others } \\
P \text { value }\end{array}$ & $\begin{array}{c}\text { Basal1- } \\
\text { HER2+ vs } \\
\text { HER2+ } \\
P \text { value*a }\end{array}$ & $\begin{array}{c}\text { Basal2- } \\
\text { HER+ vs } \\
\text { HER2+ } \\
P \text { value*a }\end{array}$ \\
\hline \multicolumn{9}{|l|}{$\begin{array}{l}\text { First clinically relevant } \\
\text { event }\end{array}$} \\
\hline Local recurrence & $1(1.1)$ & $1(8.3)$ & $1(8.3)$ & $1(2.6)$ & $\begin{array}{l}0.539 / \\
0.451 * 2\end{array}$ & $\begin{array}{c}<0.001 / \\
<0.001 * 2 * \mathrm{~b}\end{array}$ & $\begin{array}{l}0.800 / \\
0.911^{* 2}\end{array}$ & $\begin{array}{l}0.012 / \\
0.008^{* 2}\end{array}$ \\
\hline Distant metastasis & $11(12.4)$ & $2(16.7)$ & $5(41.7)$ & $7(18.4)$ & & & & \\
\hline $\begin{array}{l}\text { Contralateral breast } \\
\text { cancer }\end{array}$ & $0(0.0)$ & $0(0.0)$ & $2(16.7)$ & $0(0.0)$ & & & & \\
\hline Second primary tumor & $5(5.6)$ & $0(0.0)$ & $1(8.3)$ & $2(5.3)$ & & & & \\
\hline Not occurred & $58(65.2)$ & $7(58.3)$ & $2(16.7)$ & $24(63.2)$ & & & & \\
\hline Unknown & $8(9.0)$ & $1(8.3)$ & $0(0.0)$ & $1(2.6)$ & & & & \\
\hline $\begin{array}{l}\text { Death from other } \\
\text { causes }\end{array}$ & $6(6.7)$ & $1(8.3)$ & $1(8.3)$ & $3(7.9)$ & & & & \\
\hline \multicolumn{9}{|l|}{ First metastases site } \\
\hline Not occured & $79(88.8)$ & $9(75.0)$ & $8(66.7)$ & $33(84.6)$ & 0.155 & 0.073 & 0.566 & 0.231 \\
\hline Visceral & $3(3.4)$ & $0(0.0)$ & $2(16.7)$ & $1(2.6)$ & & & & \\
\hline No visceral & $4(4.5)$ & $1(8.3)$ & $1(8.3)$ & $3(7.7)$ & & & & \\
\hline Both & $3(3.4)$ & $2(16.7)$ & $1(8.3)$ & $2(5.1)$ & & & & \\
\hline
\end{tabular}

*2 excludes unknown category

*1 Parametric test: Independent two-sample Student's $t$ test

*a Fisher's exact test

${ }^{* b}$ Chi-square test

\section{Overall survival (OS)}

The 5-year Kaplan-Meier estimate of OS was $69 \%$ for patients with basal-HER2+ tumors, as compared with $82 \%$ for patients with luminal-HER2+ and $78 \%$ for HER2 + tumors (Figure 2). No statistical differences were found in 5-year estimated OS between patients with luminal-HER2+ tumors and those with HER2+ tumors. Upon stratification of basal-HER2+ to underlying CK5/6 expression, the 5-year Kaplan-Meier estimate of OS was $54 \%$ for patients with basal 2-HER2 + tumors (Figure 2), whereas the estimate of OS in patients with basal 1-HER2+ tumors $(82 \%)$ was not statistically different to CK-negative (luminal-HER2+ and HER2+) HER2+ patients.

We utilized Cox's proportional-hazards regression model to assess OS (Table 3). Univariate analysis revealed that the presence of the basal-like phenotype was a significant predictor of a worse 5-year OS (hazard ratio $2.36,95 \%$ confidence interval $1.18-4.75 ; P=0.0159$ ). Upon stratification of basal CK5/6 expression pattern, univariate analysis revealed that a positive $\mathrm{CK} 5 / 6$ staining in $\geq 10 \%$ of the HER $2+$ breast cancer tissue sections (but not a positive CK5/6 $<10 \%$ ) predicted a significantly worse prognosis in terms of 5-year OS (hazard ratio 4.07,
95\% confidence interval 1.88-8.79; $P<0.001)$. Neither ER nor nodal statuses were statistically significant prognostic factors of 5-year OS. When variables correlating with univariate survival at $p<0.20$ were later included in multivariate Cox regression analysis (Table 3 ), the basallike phenotype lost its independent value for predicting OS. In multivariate analysis, only age at diagnosis and tumor size remained independent predictors of OS.

\section{Disease-free survival (DFS)}

The 5-year Kaplan-Meier estimate of DFS was 52\% for patients with basal-HER2 + tumors, as compared with $77 \%$ for patients with luminal-HER $2+$, and $68 \%$ for HER 2 + tumors (Figure 3). No statistical differences were found in 5-year estimated DFS between patients with luminalHER2+ tumors and patients with HER2+. Notably, upon stratification of basal-HER2+ to underlying CK5/6 expression pattern, the 5-year Kaplan-Meier estimate of DFS was as low as $27 \%$ for patients with basal 2-HER2+ tumors (Figure 3), whereas the estimate of DFS in patients with basal 1-HER2+ tumors (75\%) was not statistically different to those with basal CK-negative (luminal-HER2+ and HER2+) HER 2+ patients. 

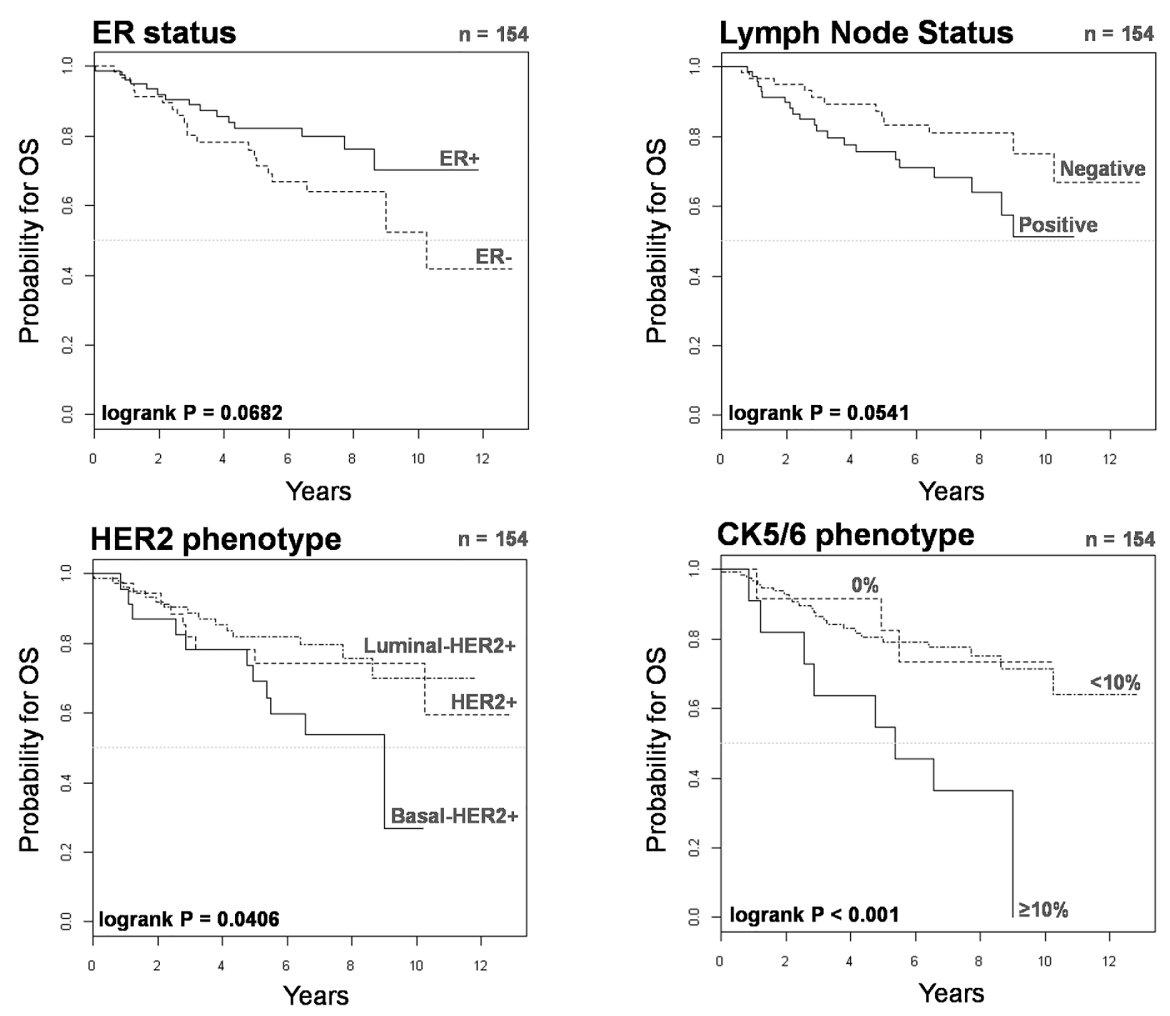

Figure 2: Kaplan-Meier OS curves of HER2+ patients stratified by ER status, lymph node status, HER2 phenotype, and $\mathrm{CK5} / 6$ phenotype.

Table 3: Cox regression analysis of factors predicting OS

\begin{tabular}{|c|c|c|c|c|}
\hline \multirow[t]{2}{*}{ Characteristics } & \multicolumn{2}{|l|}{ Univariate analysis } & \multicolumn{2}{|l|}{ Multivariate analysis $* 1$} \\
\hline & $\begin{array}{c}\text { Hazard ratio } \\
(95 \% \text { confidence interval) }\end{array}$ & $\boldsymbol{P}$ & $\begin{array}{c}\text { Hazard ratio } \\
\text { (95\% confidence interval) }\end{array}$ & $P$ \\
\hline Age (continuous) & $1.07(1.04-1.10)$ & $<0.001$ & $1.06(1.03-1.09)$ & $<0.001$ \\
\hline \multicolumn{5}{|l|}{ Tumor size } \\
\hline $\mathrm{T} 1$ & 1 & & 1 & \\
\hline $\mathrm{T} 2$ & $2.24(0.81-6.25)$ & 0.122 & $1.87(0.66-5.32)$ & 0.239 \\
\hline $\mathrm{T} 3+\mathrm{T} 4$ & $7.84(2.68-22.88)$ & $<0.001$ & $5.75(1.92-17.24)$ & 0.002 \\
\hline Inflammatory & $5.09(1.19-21.69)$ & 0.028 & $5.51(1.29-23.60)$ & 0.022 \\
\hline \multicolumn{5}{|l|}{ Lymph Node status } \\
\hline Negative & 1 & & & \\
\hline Positive & $2.01(0.97-4.14)$ & 0.059 & & \\
\hline \multicolumn{5}{|l|}{ Tumor grade } \\
\hline $1+2$ & 1 & & & \\
\hline 3 & $1.61(0.68-3.81)$ & 0.279 & & \\
\hline \multicolumn{5}{|l|}{ ER status } \\
\hline Negative & 1 & & & \\
\hline Positive & $0.54(0.28-1.06)$ & 0.072 & & \\
\hline
\end{tabular}

(Continued) 


\begin{tabular}{|c|c|c|c|c|}
\hline \multirow[t]{2}{*}{ Characteristics } & \multicolumn{2}{|l|}{ Univariate analysis } & \multicolumn{2}{|l|}{ Multivariate analysis *1 } \\
\hline & $\begin{array}{c}\text { Hazard ratio } \\
(\mathbf{9 5 \%} \text { confidence interval) }\end{array}$ & $P$ & $\begin{array}{c}\text { Hazard ratio } \\
(95 \% \text { confidence interval) }\end{array}$ & $P$ \\
\hline \multicolumn{5}{|l|}{ HER2+ phenotype } \\
\hline Luminal-HER2+ & 1 & & & \\
\hline HER $2+$ & $1.25(0.54-2.86)$ & 0.600 & & \\
\hline Basal-HER2+ & $2.55(1.19-5.46)$ & 0.016 & & \\
\hline \multicolumn{5}{|l|}{ Basal phenotype } \\
\hline Absent & 1 & & & \\
\hline Present & $2.36(1.18-4.75)$ & 0.0159 & & \\
\hline \multicolumn{5}{|l|}{ CK5/6 phenotype } \\
\hline $0 \%$ & 1 & & & \\
\hline$<10 \%$ & $1.05(0.31-3.49)$ & 0.942 & & \\
\hline$\geq 10 \%$ & $4.07(1.88-8.79)$ & $<0.001$ & & \\
\hline \multicolumn{5}{|l|}{ HER2+ Subtype } \\
\hline Luminal-HER2+ & 1 & & & \\
\hline HER 2+ & $1.25(0.54-2.86)$ & 0.599 & & \\
\hline Basal 1-HER2+ $(<10 \%)$ & $1.13(0.33-3.91)$ & 0.849 & & \\
\hline Basal 2-HER2+ $(\geq 10 \%)$ & $4.39(1.91-10.05)$ & $<0.001$ & & \\
\hline \multicolumn{5}{|l|}{ Adjuvant trastuzumab } \\
\hline No & 1 & & & \\
\hline Yes & $0.87(0.43-1.80)$ & 0.714 & & \\
\hline \multicolumn{5}{|l|}{ Adjuvant chemotherapy } \\
\hline No & 1 & & & \\
\hline Yes & $0.82(0.38-1.78)$ & 0.615 & & \\
\hline \multicolumn{5}{|l|}{ Adjuvant hormone therapy } \\
\hline No & 1 & & & \\
\hline Yes & $0.37(0.18-0.77)$ & 0.007 & & \\
\hline
\end{tabular}

*1 proportional hazard assumption for the Cox model has been checked

We utilized Cox's proportional-hazards regression model to assess DFS (Table 4). Univariate analysis revealed that the presence of the basal-like phenotype was a significant predictor of a worse 5-year DFS (hazard ratio $2.05,95 \%$ confidence interval 1.04-4.04; $P=0.037$ ). Upon stratification of basal CK5/6 expression pattern, univariate analysis revealed that a positive CK5/6 staining in $\geq 10 \%$ of the HER $2+$ breast cancer tissue sections (but not a positive CK $5 / 6<10 \%$ ) predicted a significantly worse 5-year DFS (hazard ratio 4.17, 95\% confidence interval $1.96-8.98 ; P<0.001)$. Neither ER nor nodal statuses were statistically significant prognostic factors of 5-year DFS. Importantly, when variables correlating with univariate survival at $p<0.20$ were later included in multivariate Cox regression analysis (Table 4), the presence of the basal 2-HER2+ phenotype (i.e., a positive CK5/6 staining in more than $10 \%$ of the HER $2+$ breast cancer tissue sections) retained its independent value for predicting a worse outcome in terms of DFS (hazard ratio $2.44,95 \%$ confidence interval $1.05-5.67 ; P=0.037$ ). In multivariate analysis, tumor size also remained an independent predictor of a worse DFS.

\section{Time to treatment failure (TTF)}

We investigated the prognostic significance of the basal-HER2+ phenotype in 104 HER2+ patients treated with either chemotherapy-only or trastuzumab-based 

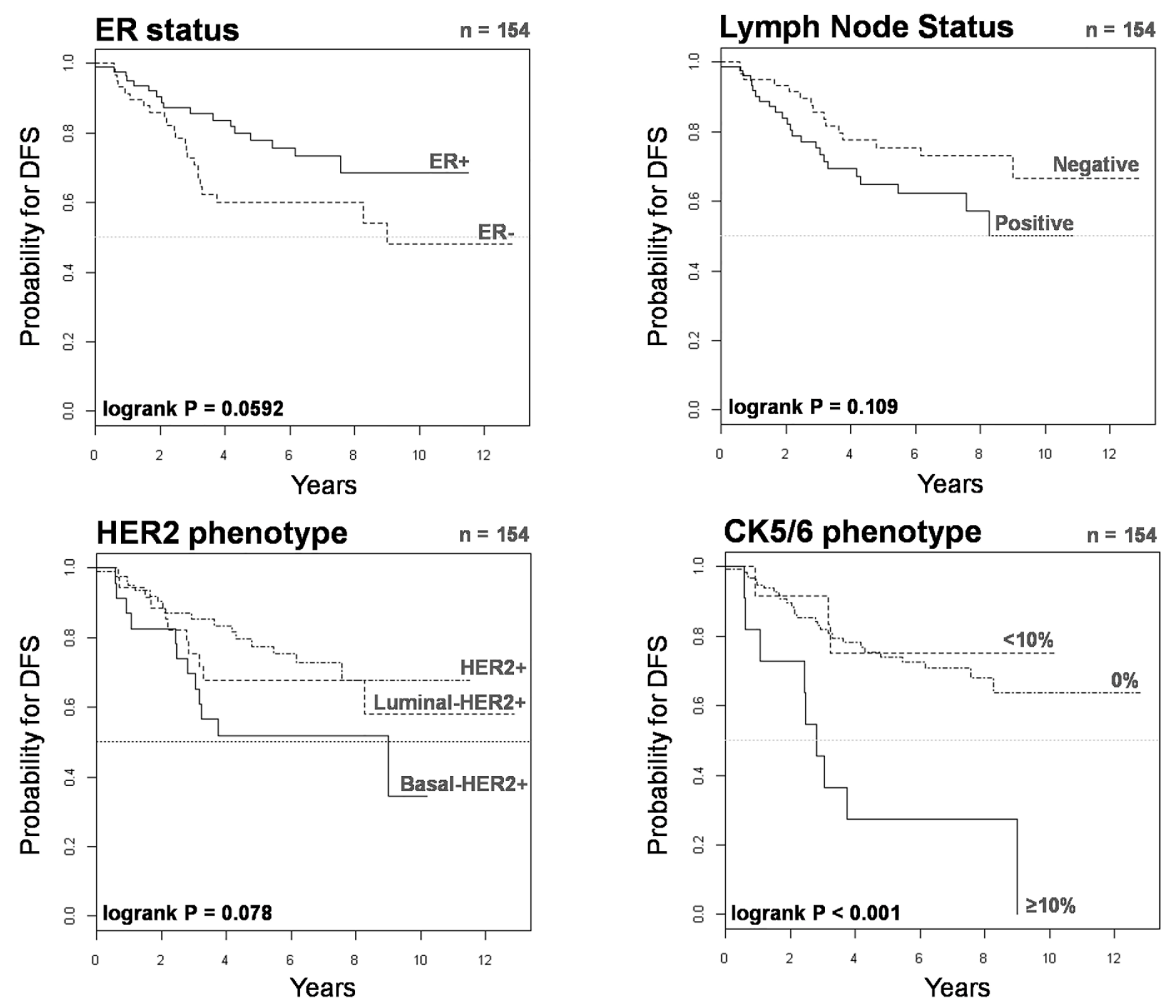

Figure 3: Kaplan-Meier DFS curves of HER2+ patients stratified by ER status, lymph node status, HER2 phenotype, and $\mathrm{CK5} / 6$ phenotype.

\section{Table 4: Cox regression analysis of factors predicting DFS}

\begin{tabular}{|c|c|c|c|c|}
\hline \multirow[t]{3}{*}{ Characteristics } & \multicolumn{2}{|c|}{ Univariate analysis } & \multicolumn{2}{|c|}{ Multivariate analysis *1 } \\
\hline & \multirow{2}{*}{\multicolumn{2}{|c|}{$\begin{array}{c}\text { Hazard ratio } \\
(95 \% \text { confidence interval) }\end{array}$}} & \multirow{2}{*}{\multicolumn{2}{|c|}{$\begin{array}{c}\text { Hazard ratio } \\
(95 \% \text { confidence interval) }\end{array}$}} \\
\hline & & & & \\
\hline Age (continuous) & $1.02(1.00-1.04)$ & 0.115 & & \\
\hline \multicolumn{5}{|l|}{ Tumor size } \\
\hline $\mathrm{T} 1$ & 1 & & 1 & \\
\hline $\mathrm{T} 2$ & $3.38(1.27-9.02)$ & 0.015 & $3.05(1.13-8.23)$ & 0.028 \\
\hline $\mathrm{T} 3+\mathrm{T} 4$ & $7.74(2.64-22.71)$ & $<0.001$ & $6.41(2.05-20.05)$ & 0.001 \\
\hline Inflammatory & $5.21(1.23-22.00)$ & 0.025 & $5.17(1.21-21.98)$ & 0.026 \\
\hline \multicolumn{5}{|l|}{ Node status } \\
\hline Negative & 1 & & & \\
\hline Positive & $1.69(0.88-3.23)$ & 0.113 & & \\
\hline \multicolumn{5}{|l|}{ Tumor grade } \\
\hline $1+2$ & 1 & & & \\
\hline 3 & $1.01(0.48-2.10)$ & 0.989 & & \\
\hline \multicolumn{5}{|l|}{ ER status } \\
\hline Negative & 1 & & & \\
\hline Positive & $0.55(0.29-1.03)$ & 0.063 & & \\
\hline
\end{tabular}

(Continued) 


\begin{tabular}{|c|c|c|c|c|}
\hline \multirow[t]{2}{*}{ Characteristics } & \multicolumn{2}{|l|}{ Univariate analysis } & \multicolumn{2}{|l|}{ Multivariate analysis *1 } \\
\hline & $\begin{array}{c}\text { Hazard ratio } \\
(95 \% \text { confidence interval) }\end{array}$ & $P$ & $\begin{array}{c}\text { Hazard ratio } \\
(95 \% \text { confidence interval) }\end{array}$ & $P$ \\
\hline \multicolumn{5}{|l|}{ HER2+ phenotype } \\
\hline Luminal-HER2+ & 1 & & & \\
\hline HER2+ & $1.37(0.64-2.93)$ & 0.416 & & \\
\hline Basal-HER2+ & $2.29(1.10-4.82)$ & 0.028 & & \\
\hline \multicolumn{5}{|l|}{ Basal phenotype } \\
\hline Absent & 1 & & & \\
\hline Present & $2.05(1.04-4.04)$ & 0.037 & & \\
\hline \multicolumn{5}{|l|}{ CK5/6 phenotype } \\
\hline $0 \%$ & 1 & 1 & & \\
\hline$<10 \%$ & $0.81(0.25-2.68)$ & 0.734 & $0.60(0.18-2.02)$ & 0.409 \\
\hline$\geq 10 \%$ & $4.17(1.96-8.87)$ & $<0.001$ & $2.44(1.05-5.67)$ & 0.037 \\
\hline \multicolumn{5}{|l|}{ HER2+ Subtype } \\
\hline Luminal-HER2+ & 1 & & & \\
\hline HER 2+ & $1.37(0.64-2.93)$ & 0.415 & & \\
\hline Basal 1-HER2+ $(<10 \%)$ & $0.91(0.27-3.11)$ & 0.880 & & \\
\hline Basal 2-HER2+ $(\geq 10 \%)$ & $4.67(2.08-10.51)$ & $<0.001$ & & \\
\hline \multicolumn{5}{|l|}{ Adjuvant trastuzumab } \\
\hline No & 1 & & & \\
\hline Yes & $0.98(0.51-1.87)$ & 0.953 & & \\
\hline \multicolumn{5}{|l|}{ Adjuvant chemotherapy } \\
\hline No & 1 & & & \\
\hline Yes & $0.82(0.40-1.70)$ & 0.590 & & \\
\hline \multicolumn{5}{|l|}{ Adjuvant hormone therapy } \\
\hline No & 1 & & & \\
\hline Yes & $0.60(0.32-1.13)$ & 0.111 & & \\
\hline
\end{tabular}

*1 proportional hazard assumption for the Cox model has been checked

adjuvant $(n=67,64 \%) /$ neoadjuvant $(n=37,36 \%)$ chemotherapy. We utilized Cox's proportional-hazards regression model to assess whether the basal-HER2+ might constitute a predictor of a worse course in terms of time to treatment failure (TTF) (Table 5). Univariate analysis revealed that tumor size, node status and the presence of a basal-like phenotype predicted a significantly worsened TTF (Figure 4). Upon stratification of basal CK5/6 expression pattern, univariate analysis revealed that a positive CK5/6 staining in $\geq 10 \%$ of the HER $2+$ breast cancer tissue sections (but not a positive CK5/6 $<10 \%$ ) was a significant predictor of worse outcome in terms of TTF (hazard ratio 5.45, 95\% confidence interval
2.07-14.35; $P<0.001)$. Remarkably, after accounting for prognostic variables correlating with univariate survival at $p<0.20$ (Table 5), tumor size and the presence of the basal 2-HER2+ phenotype (i.e., a positive CK5/6 staining in $\geq$ $10 \%$ of the HER $2+$ breast cancer tissue sections) retained their independent value for predicting a worse prognosis in terms of TTF (hazard ratio 3.66; 95\% confidence interval 1.24-10.78; $P=0.019$ ) in multivariate Cox regression analysis.

We finally assessed the prognostic significance of the basal-HER $2+$ phenotype in 69 HER $2+$ patients treated with trastuzumab-based adjuvant $(n=42,61 \%) /$ neoadjuvant $(n=27,39 \%)$ therapy (Table 6). Upon 
Table 5: Cox regression analysis of factors predicting TTF

\begin{tabular}{|c|c|c|c|c|}
\hline \multirow[t]{3}{*}{ Characteristics } & \multicolumn{2}{|l|}{ Univariate analysis } & \multicolumn{2}{|l|}{ Multivariate analysis $* 1$} \\
\hline & Hazard ratio & $\boldsymbol{P}$ & Hazard ratio & $\boldsymbol{P}$ \\
\hline & (95\% confidence interval) & & (95\% confidence interval) & \\
\hline Age (continuous) & $1.01(0.98-1.05)$ & 0.384 & & \\
\hline \multicolumn{5}{|l|}{ Tumor size } \\
\hline $\mathrm{T} 1$ & 1 & & 1 & \\
\hline $\mathrm{T} 2$ & $7.74(1.01-59.55)$ & 0.049 & $6.73(0.87-52.25)$ & 0.068 \\
\hline $\mathrm{T} 3+\mathrm{T} 4$ & $19.80(2.38-165.03)$ & 0.006 & $11.64(1.29-104.96)$ & 0.029 \\
\hline Inflammatory & $16.03(1.65-155.62)$ & 0.017 & $15.09(1.54-147.66)$ & 0.020 \\
\hline \multicolumn{5}{|l|}{ Node status } \\
\hline Negative & 1 & & & \\
\hline Positive & $3.42(1.16-10.08)$ & 0.026 & & \\
\hline \multicolumn{5}{|l|}{ Tumor grade } \\
\hline $1+2$ & 1 & & & \\
\hline 3 & $0.90(0.31-2.55)$ & 0.836 & & \\
\hline \multicolumn{5}{|l|}{ ER status } \\
\hline Negative & 1 & & & \\
\hline Positive & $0.56(0.24-1.29)$ & 0.170 & & \\
\hline \multicolumn{5}{|l|}{ HER2+ phenotype } \\
\hline Luminal-HER2+ & 1 & & & \\
\hline HER2+ & $1.11(0.37-3.33)$ & 0.848 & & \\
\hline Basal-HER2+ & $2.72(1.08-6.87)$ & 0.034 & & \\
\hline \multicolumn{5}{|l|}{ Basal phenotype } \\
\hline Absent & 1 & & & \\
\hline Present & $2.63(1.14-6.07)$ & 0.024 & & \\
\hline \multicolumn{5}{|l|}{ CK5/6 phenotype } \\
\hline $0 \%$ & 1 & & 1 & \\
\hline$<10 \%$ & $1.30(0.37-4.52)$ & 0.684 & $1.26(0.36-4.47)$ & 0.717 \\
\hline$\geq 10 \%$ & $5.45(2.07-14.35)$ & $<0.001$ & $3.66(1.24-10.78)$ & 0.019 \\
\hline \multicolumn{5}{|l|}{ HER2+ Subtype } \\
\hline Luminal-HER2+ & 1 & & & \\
\hline HER2+ & $1.11(0.37-3.32)$ & 0.855 & & \\
\hline Basal 1-HER2+ $(<10 \%)$ & $1.34(0.36-4.97)$ & 0.659 & & \\
\hline Basal 2-HER2+ $(\geq 10 \%)$ & $5.64(1.99-15.99)$ & 0.001 & & \\
\hline \multicolumn{5}{|l|}{ Treatment } \\
\hline Only Chemotherapy & 1 & & & \\
\hline $\begin{array}{l}\text { Trastuzumab }+ \\
\text { Chemotherapy }\end{array}$ & $0.99(0.43-2.30)$ & 0.989 & & \\
\hline
\end{tabular}

\footnotetext{
${ }^{* 1}$ proportional hazard assumption for the Cox model has been checked
} 

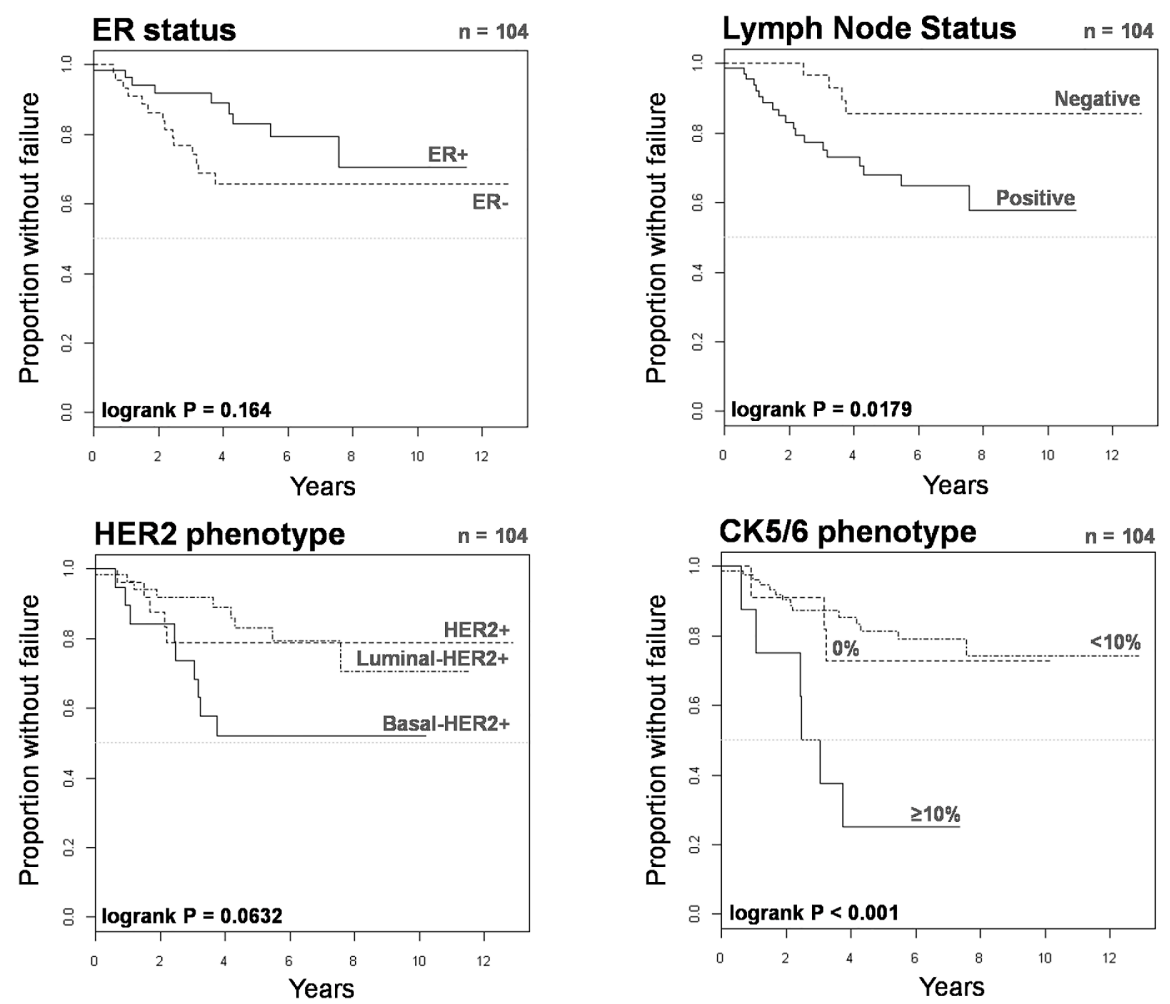

Figure 4: Kaplan-Meier TTF curves of HER2+ patients treated with chemotherapy only or trastuzumab-based therapy when stratified by ER status, lymph node status, HER2 phenotype, and CK5/6 phenotype.

Table 6: Cox regression analysis of factors predicting time to trastuzumab failure

\begin{tabular}{|c|c|c|c|c|}
\hline \multirow[t]{3}{*}{ Characteristics } & \multicolumn{2}{|l|}{ Univariate analysis } & \multicolumn{2}{|l|}{ Multivariate analysis $* 1$} \\
\hline & Hazard ratio & $P$ & Hazard ratio & $P$ \\
\hline & ( $95 \%$ confidence interval) & & ( $95 \%$ confidence interval) & \\
\hline Age (continuous) & $1.03(0.98-1.08)$ & 0.264 & & \\
\hline \multicolumn{5}{|l|}{ Tumor size } \\
\hline $\mathrm{T} 1$ & 1 & & & \\
\hline $\mathrm{T} 2$ & $4.72(0.57-39.34)$ & 0.152 & & \\
\hline $\mathrm{T} 3+\mathrm{T} 4$ & $14.19(1.43-140.77)$ & 0.024 & & \\
\hline Inflammatory & $7.05(0.63-78.70)$ & 0.113 & & \\
\hline \multicolumn{5}{|l|}{ Node status } \\
\hline Negative & - & - & & \\
\hline Positive & - & - & & \\
\hline \multicolumn{5}{|l|}{ Tumor grade } \\
\hline $1+2$ & 1 & & & \\
\hline 3 & $1.27(0.33-4.92)$ & 0.731 & & \\
\hline \multicolumn{5}{|l|}{ ER status } \\
\hline Negative & 1 & & & \\
\hline Positive & $0.65(0.22-1.95)$ & 0.447 & & \\
\hline
\end{tabular}

(Continued) 


\begin{tabular}{|c|c|c|c|c|}
\hline \multirow[t]{3}{*}{ Characteristics } & \multicolumn{2}{|l|}{ Univariate analysis } & \multicolumn{2}{|l|}{ Multivariate analysis $* 1$} \\
\hline & Hazard ratio & $\boldsymbol{P}$ & Hazard ratio & $P$ \\
\hline & ( $95 \%$ confidence interval) & & ( $95 \%$ confidence interval) & \\
\hline \multicolumn{5}{|l|}{ HER2+ phenotype } \\
\hline Luminal-HER2+ & 1 & & & \\
\hline HER2+ & $1.29(0.32-5.18)$ & 0.724 & & \\
\hline Basal-HER2+ & $1.79(0.49-6.46)$ & 0.376 & & \\
\hline \multicolumn{5}{|l|}{ Basal phenotype } \\
\hline Absent & 1 & & & \\
\hline Present & $1.66(0.50-5.54)$ & 0.408 & & \\
\hline \multicolumn{5}{|l|}{ CK5/6 phenotype } \\
\hline $0 \%$ & 1 & & 1 & \\
\hline$<10 \%$ & $0.89(0.18-4.43)$ & 0.888 & $0.89(0.18-4.43)$ & 0.888 \\
\hline$\geq 10 \%$ & $6.80(1.39-33.36)$ & 0.018 & $6.80(1.39-33.36)$ & 0.018 \\
\hline \multicolumn{5}{|l|}{ HER2+ Subtype } \\
\hline Luminal-HER2+ & 1 & & & \\
\hline HER 2+ & $1.34(0.33-5.43)$ & 0.684 & & \\
\hline Basal 1-HER2+ $(<10 \%)$ & $0.96(0.19-4.97)$ & 0.966 & & \\
\hline Basal 2-HER2+ $(\geq 10 \%)$ & $7.49(1.41-39.70)$ & 0.018 & & \\
\hline
\end{tabular}

${ }^{* 1}$ proportional hazard assumption for the Cox model has been checked

stratification of basal CK5/6 expression pattern, univariate analysis confirmed that a positive CK5/6 staining in $\geq$ $10 \%$ of the HER $2+$ breast cancer tissue sections (but not a positive CK5/6 $<10 \%$ ) was a significant predictor of worse outcome in terms of TTF in trastuzumab-treated patients (hazard ratio 7.49, 95\% confidence interval 1.4139.70; $P=0.018$ ). More importantly, a positive CK5/6 staining in $\geq 10 \%$ of the HER2+ breast cancer tissue sections remained the sole independent factor predicting a worse outcome of trastuzumab-treated patients in terms of TTF in multivariate Cox regression analysis (hazard ratio $6.80,95 \%$ confidence interval $1.39-33.36$ ). KaplanMeier estimates of TTF confirmed that patients with basal 2-HER2+ tumors did not benefit from adding trastuzumab to chemotherapy (Figure 5).

\section{DISCUSSION}

Currently available gene expression signatures cannot identify HER2+ subgroups of good- and poorresponders to trastuzumab. In an ambitious attempt to address the need for a predictor of trastuzumab benefit, Pogue-Geile et al. [8] developed an eight-gene model able to stratify HER2+ tumors into a discontinuous distribution of patients within 3 different subsets of "extraordinary", "moderate", and "no apparent" benefit from trastuzumab.
This complexity makes the establishment of optimal cutoffs nearly impossible and the validation of the eight-gene classifier, if applied arbitrarily, could lead to misclassification of HER2+ patients and thus inappropriate treatment recommendations [22]. Using molecular profiling to investigate the variable prognosis and response to therapy of HER2+ tumors, Staaf et al. [23] identified a 158-gene HER2-derived prognostic predictor (HDPP) that improved the stratification of good and poor prognosis for both OS and distant metastasis-free survival in both the HER2-enriched molecular subtype and the basal-like subtype, which are assumed to be mutually exclusive breast cancer entities. When applied to a small HER2+ group of patients preoperatively treated with trastuzumab, the HDPP signature correlated with the occurrence of trastuzumab resistance. Although the sample size was insufficient to form any conclusion, it should be noted that when formerly analyzing this data set, Harris et al. [9] posited that "HER2-overexpressing tumors with a basal-like phenotype" were more likely to be intrinsically resistant to pre-operative trastuzumab. Here, we present evidence that immunohistochemical reclassification of HER2+ breast cancer tumors by basal CK5/6 expression might be sufficient to significantly improve prognosis and trastuzumab-based therapeutic planning. 

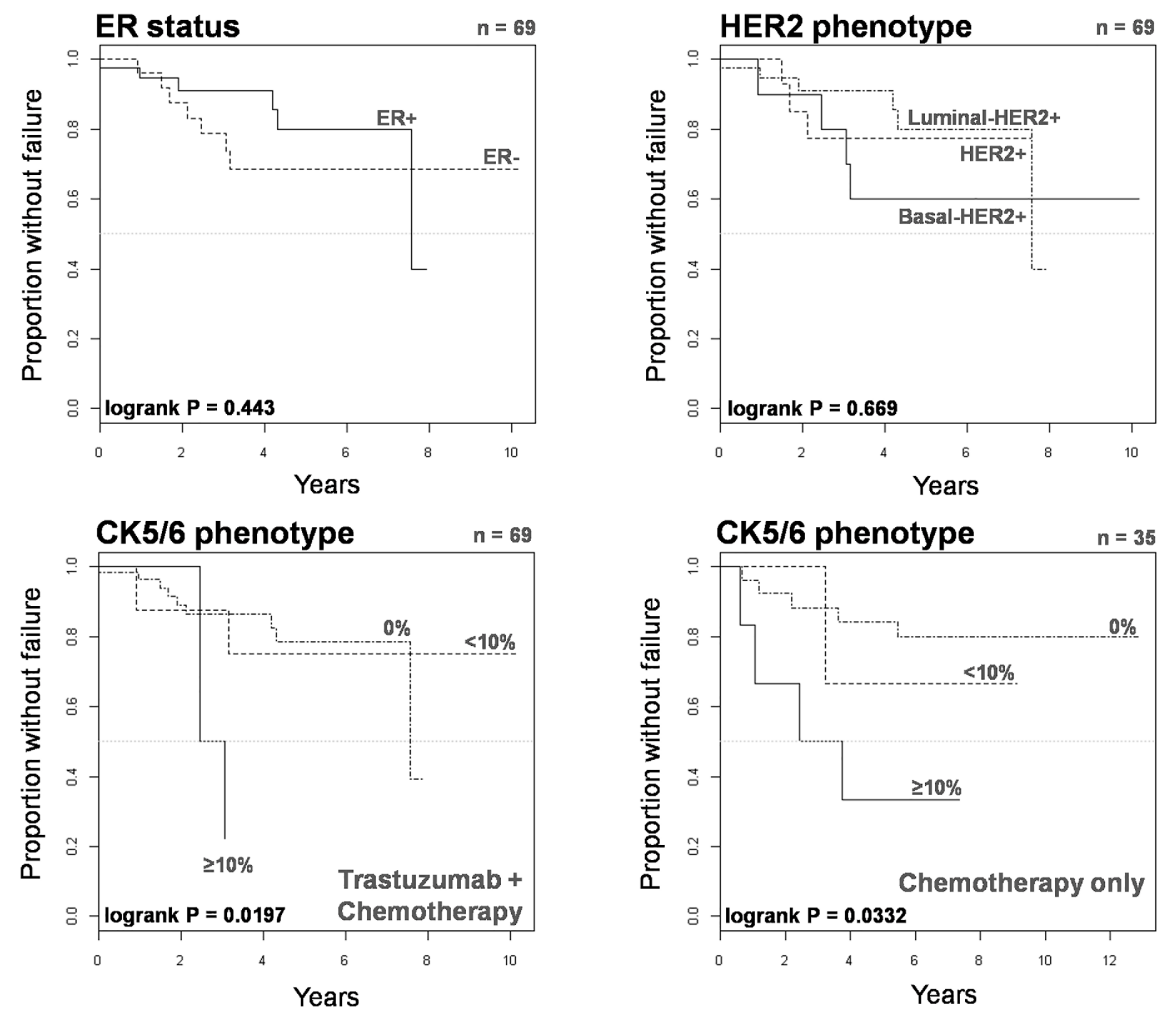

Figure 5: Kaplan-Meier TTF curves of HER2+ patients treated with trastuzumab-based therapy when stratified by ER status, HER2 phenotype, and CK5/6 phenotype.

Previous morpho-immunohistologic studies [15-19] have shown that a subgroup of HER2+ tumors consistently demonstrate several features classically ascribed to basal-like tumors, including poor differentiation, highgrade, geographic necrosis, pushing margins of invasion, syncytial arrangement of tumor cells, ribbon- or festoonlike, squamous metaplasia, stromal lymphocytic infiltrates, high mitotic index, and p53 positivity. Importantly, these basal-HER2+ breast carcinomas show immunoreactivity for the basal epithelium cytokeratin marker CK5/6, which at times appears as a uniform or almost uniform positive staining that fully mimics the pattern of pure basal-like breast carcinomas, but often displays checkerboard-type intratumoral heterogeneity. Here we confirm that the basalHER2+ phenotype defined by the immunohistochemical expression of basal CK5/6 is associated with aggressive disease and adversely impacts survival in HER2+ breast cancer patients. In our series, there were no statistically significant differences in OS and DFS between patients with ER-positive luminal-HER2+ tumors and ER-negative HER2+ tumors. However, the basal-HER2+ phenotype was significantly associated with inferior OS by univariate analysis and, after accounting for strong prognostic variables such as tumor size at diagnosis in stepwise multivariate analysis, the presence of the basal-HER2+ phenotype, but not ER status, also predicted significantly worsened DFS when a positive CK5/6 staining in $\geq 10 \%$ of the HER2+ breast cancer tissue sections was registered as a diagnostically relevant positive reaction.

Our findings might not only have important implications for prognosis, but also for therapy. The simple and specific $\mathrm{CK} 5 / 6$ fingerprint using a $10 \%$ cutoff allows the re-classification of HER2+ tumors into a sub-class of basal 1-HER $2+$ tumors $(<10 \%$ of cells showing positive CK5/6 staining), which appear to be prognostically indistinguishable from HER2+ tumors, and a sub-class of clinically aggressive basal 2 -HER $2+$ tumors $(\geq 10 \%$ of cells showing positive CK5/6 staining) which will likely be unresponsive to trastuzumab-based adjuvant/ neoadjuvant therapy. While acknowledging that our study is small and exploratory and the data on adjuvant/ neoadjuvant trastuzumab are limited in size (approximately $45 \%$ of HER $2+$ patients) and maturity (median follow-up is approximately 4 years for patients treated with adjuvant trastuzumab), the fact that the use of adjuvant/neoadjuvant trastuzumab and chemotherapy did not greatly differ between patients with HER2+ and basal 2-HER2+ tumors together with the strong association between the basal 2-HER2+ phenotype and worsened survival and shorter time to trastuzumab-based treatment failure, strongly suggest that immunohistochemical identification of the basal 2-HER2+ phenotype may be used as a predictive marker of primary refractoriness to trastuzumab. Accordingly, the co-expression of well-known basal-like molecular features, 
including expression of the anti-apoptotic protein survivin, the dynamic emergence of the $\mathrm{CD} 44^{+} \mathrm{CD} 24^{- \text {/low }}$ breast cancer stem cell (CSC) immunophenotype, or the occurrence of epithelial-to-mesenchymal transition (EMT) phenomena, occurs exclusively in HER2+ breast cancer cells exhibiting primary resistance to trastuzumab [10, 19, 24-30].

The a priori characterization of distinct biological HER2+ breast cancer subgroups associated with poorer prognosis and resistance to trastuzumab-based adjuvant/ neoadjuvant therapy using DNA microarrays is not currently feasible for large-scale clinical applications. In this setting, immunohistochemical staining of basal CK5/6 can be a useful surrogate to predict inferior survival and poorer responses to trastuzumab-based therapy. In our hands, a CK5/6-based fingerprint using a $10 \%$ positivity cutoff allows the rapid re-classification of HER2+ tumors in a manner that improves prognosis and therapeutic planning in a sub-class of patients with clinically aggressive basal-HER2+ tumors who are unlikely to benefit from trastuzumab-based adjuvant therapy (Figure 6). It is tempting to suggest that CK5/6-defined basal-HER2 + tumors might be viewed as an immunohistochemical algorithm, analogous to EMT and CSC-like gene signatures and likely contributes to the poor outcomes in basal-HER2+ tumors [31-36]. Although larger retrospective studies should be conducted to unequivocally determine whether basal-HER2+ tumors are differentially enriched with cells combining EMT/CSC phenotypes, it is noteworthy that the sole re-classification of ER-/HER2 + tumors by the expression of the core EMT transcription factors SNAI2 (SLUG) and TWIST [37] using the on-line Kaplan-Meier plotter (http://kmplot. $\mathrm{com} /$ ) [38, 39], was sufficient to predict a significantly inferior relapse free survival (RFS) and distant metastasis free survival (DMFS) in the EMT-like/HER2+ patient cohort (Figure 7).

There is an urgent need to generate a more definitive taxonomy of HER2+ breast carcinomas as well as molecular forecasting signatures to be validated prospectively and in samples from trastuzumab-based clinical trials. Our current findings lend support to forthcoming prospective studies aimed to validate the concept that identification of CK5/6-positive immunophenotypes within HER2+ breast carcinomas may be a rapid and accurate method for identifying intrinsic subgroups of biologically aggressive tumors likely to display resistance to trastuzumab $a b$ initio in women diagnosed with HER2+ cancer.

\section{METHODS}

\section{Study patients}

The prospectively maintained breast cancer database at the Hospital Universitari de Girona Dr. Josep Trueta in Girona, Spain was queried to identify patients diagnosed with HER2-overexpressing primary breast cancer between June 2000 and February 2014. The study was limited to the most common breast cancer histology, i.e., invasive ductal cancer, to allow for more accurate prognostication. The investigators irreversibly anonymized (stripped of any link to the original patient) all the tissues and existing data in such a manner that subjects cannot be identified, directly or through identifiers linked to the subjects.

\section{Immunohistochemistry (IHC) and classification}

Primary tumor size according to pathological analysis was classified according to the Seventh Edition of the AJCC Cancer Staging Manual. A board-certified specialty-trained breast pathologist (E. L. B.) reviewed immunohistochemical (ER, HER2) stainings from 154 consecutive patients with HER2-overexpressing primary invasive ductal breast cancer. Approximately 30\% of the study cohort was previously checked for receptor expression, which was undertaken as an internal quality control measure to confirm that the ER and HER2 status of patients at the time of performance of this study was in agreement with that initially rendered at the time of diagnosis. No noticeable differences were encountered when ER and HER2 IHC staining was scored using criteria from published guidelines. The cutoff for ER immunoreactivity was $10 \%$ positive tumor nuclei irrespective of intensity. HER2 expression status was considered positive if immunostaining was scored as 3+ (i.e., strongly positive in $>10 \%$ of cancer cells by IHC) according to HercepTest criteria. For an equivocal result of HER2 $2+$ (i.e., moderately positive in $>10 \%$ of cancer cells by IHC), HER2 expression status was considered positive if fluorescent in situ hybridization (FISH) assay revealed a HER2:chromosome-17 amplification ratio of $>2.2$.

Paraffin-embedded tissue sections from HER2+ tumors were immunostained with CK5/6 (clone D5/16B4, Dako; 1:50 dilution) antibody. Antigen retrieval was performed using Tris-EDTA pH 9 for 30 minutes. Analysis was carried out by a board-certified pathologist (E. L. B.), who scored the basal CK5/6 staining on a scale of $0-2$ : 0 , no staining; $1,<10 \%$ of cells showing positive staining; and $2, \geq 10 \%$ of cells showing positive. A CK5/6 score greater than 0 originally defined a positive basal phenotype. Tumors were then classified as luminal/ HER2+ subtype (ER positive and basal CK5/6 negative), HER2+ subtype (ER negative and basal CK5/6 negative), and basal-HER2+ subtype (ER negative and basal CK5/6 positive) (Figure 1).

The current study is reported according to the Reporting Recommendations for Tumor Marker Prognostic Studies (REMARK). Laboratory personnel who were blinded to clinical data and outcomes performed all IHC assays. Assay results were interpreted and scored by a pathologist (E. L. B.) who remained blinded to clinical data. 

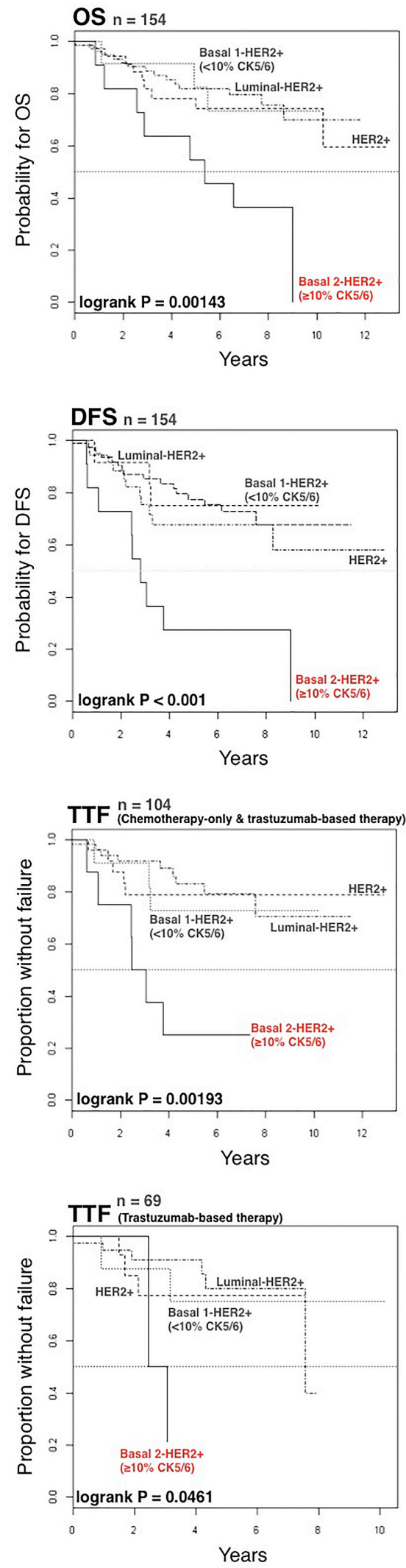

Figure 6: Kaplan-Meier OS, DFS, and TTF curves for patients with luminal-HER2+, HER2+, basal 1-HER2+ $(<\mathbf{1 0} \%$ CK5/6), and basal 2-HER2+ ( $\geq 10 \% \mathrm{CK5} / 6)$ tumors as defined by immunohistochemical analysis (Figure 1). 

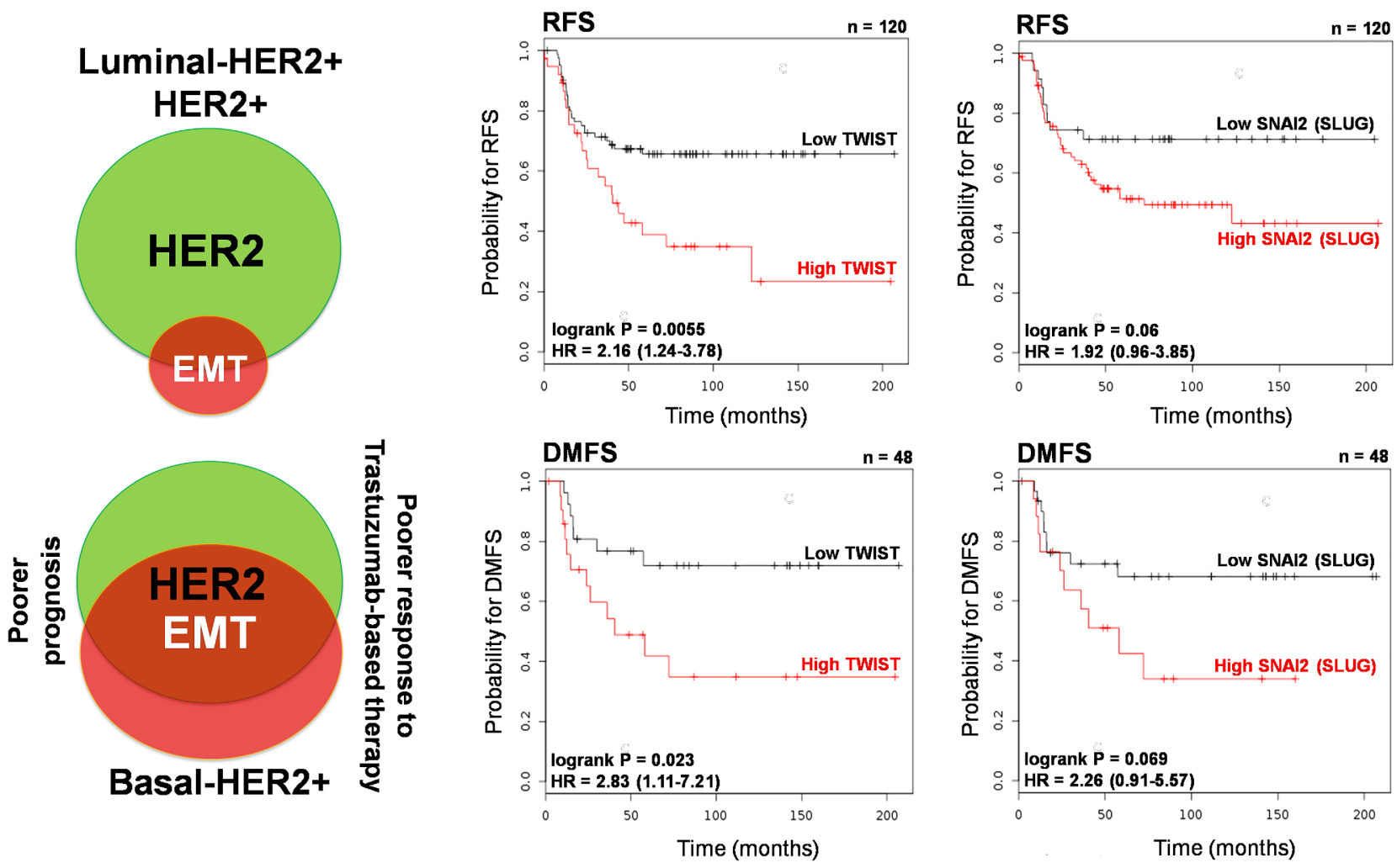

Figure 7: Kaplan-Meier RFS and DMFS curves of HER2+ patients stratified by low/high TWIST and low/high SNAI2 (SLUG) gene expression.

\section{Statistical analysis}

All statistical data analyses were performed using IBM SPSS version 21.0 (IBM Corp. 2012) and R software environment (http://www.r-project.org/). Data collected included date of birth, date of diagnosis, tumor size, tumor grade, lymph node status, receipt of adjuvant therapy (hormone, trastuzumab, and chemotherapy), site and date of recurrent disease, and date of death. Proportions observed among categorical variables were compared using the chi-squared test or Fisher's exact test. The Mann-Whitney $U$ test was used to test for differences in continuous variables. Survival functions were estimated for disease-free survival and overall survival using the product-limit method of Kaplan and Meier. Overall survival (OS) was calculated from the date of diagnosis to the date of death. Disease-free survival (DFS) was calculated from the date of initial diagnosis to the date of recurrence or death, whichever came first. Patients not experiencing an event were considered censored at the date of last contact. Time to treatment failure (TTF) was calculated from the date of therapy initiation to the time of first evidence of failure, i.e., disease progression, recurrence or death. Inference on survival functions among subgroups was based on the log-rank test for the equality of the survival functions. Cox's proportionalhazards regression model was used to identify statistically significant predictors of OS, DFS, and TTF and the proportional hazard assumption was validated. In such models, the covariate function is proportional to the hazard or mortality and, therefore, positive coefficients indicate a shorter survival with increasing value of the covariate. $P$ values of less than 0.05 were considered to be statistically significant.

\section{ACKNOWLEDGMENTS}

This work was supported by grants from the Ministerio de Ciencia e Innovación (Grant SAF201238914), Plan Nacional de I+D+I, Spain and the Agència de Gestió d'Ajuts Universitaris I de Recerca (AGAUR) (Grant 2014 SGR229), Departament d'Economia I Coneixement, Catalonia, Spain.

\section{CONFLICT OF INTEREST STATEMENT}

The authors of this manuscript have no conflicts of interest to declare. 


\section{REFERENCES}

1. Romond EH, Perez EA, Bryant J, Suman VJ, Geyer CE Jr, Davidson NE, Tan-Chiu E, Martino S, Paik S, Kaufman PA, Swain SM, Pisansky TM, Fehrenbacher L, et al. Trastuzumab plus adjuvant chemotherapy for operable HER2-positive breast cancer. N Engl J Med. 2005; 353:1673-1684.

2. Perez EA, Romond EH, Suman VJ, Jeong JH, Sledge G, Geyer CE Jr, Martino S, Rastogi P, Gralow J, Swain SM, Winer EP, Colon-Otero G, Davidson NE, et al. Trastuzumab Plus Adjuvant Chemotherapy for Human Epidermal Growth Factor Receptor 2-Positive Breast Cancer: Planned Joint Analysis of Overall Survival From NSABP B-31 and NCCTG N9831. J Clin Oncol. 2014; 32:3744-3752.

3. Perez EA, Dueck AC, McCullough AE, Reinholz MM, Tenner KS, Davidson NE, Gralow J, Harris LN, Kutteh LA, Hillman DW, Jenkins RB, Chen B. Predictability of adjuvant trastuzumab benefit in N9831 patients using the ASCO/CAP HER2-positivity criteria. J Natl Cancer Inst. 2012; 104:159-162.

4. Perez EA, Dueck AC, McCullough AE, Chen B, Geiger XJ, Jenkins RB, Lingle WL, Davidson NE, Martino S, Kaufman PA, Kutteh LA, Sledge GW, Harris LN, et al. Impact of PTEN protein expression on benefit from adjuvant trastuzumab in early-stage human epidermal growth factor receptor 2-positive breast cancer in the North Central Cancer Treatment Group N9831 trial. J Clin Oncol. 2013; 31:2115-2122.

5. Perez EA, Reinholz MM, Hillman DW, Tenner KS, Schroeder MJ, Davidson NE, Martino S, Sledge GW, Harris LN, Gralow JR, Dueck AC, Ketterling RP, Ingle JN, et al. HER2 and chromosome 17 effect on patient outcome in the N9831 adjuvant trastuzumab trial. J Clin Oncol. 2010; 28:4307-4315.

6. Moreno-Aspitia A, Hillman DW, Dyar SH, Tenner KS, Gralow J, Kaufman PA, Davidson NE, Lafky JM, Reinholz MM, Lingle WL, Kutteh LA, Carney WP, Dueck AC, et al. Soluble human epidermal growth factor receptor 2 (HER2) levels in patients with HER2-positive breast cancer receiving chemotherapy with or without trastuzumab: results from North Central Cancer Treatment Group adjuvant trial N9831. Cancer. 2013; 119:2675-2682.

7. Paik S, Kim C, Wolmark N. HER2 status and benefit from adjuvant trastuzumab in breast cancer. N Engl J Med. 2008; 358:1409-1411.

8. Pogue-Geile KL, Kim C, Jeong JH, Tanaka N, Bandos H, Gavin PG, Fumagalli D, Goldstein LC, Sneige N, Burandt E, Taniyama Y, Bohn OL, Lee A, et al. Predicting degree of benefit from adjuvant trastuzumab in NSABP trial B-31. J Natl Cancer Inst. 2013; 105:1782-1788.

9. Harris LN, You F, Schnitt SJ, Witkiewicz A, Lu X, Sgroi D, Ryan PD, Come SE, Burstein HJ, Lesnikoski BA, Kamma M, Friedman PN, Gelman R, et al. Predictors of resistance to preoperative trastuzumab and vinorelbine for HER2-positive early breast cancer. Clin Cancer Res. 2007; 13:1198-1207.

10. Martin-Castillo B, Oliveras-Ferraros C, Vazquez-Martin A, Cufí S, Moreno JM, Corominas-Faja B, Urruticoechea A, Martín ÁG López-Bonet, E Menendez. Basal/HER2 breast carcinomas: integrating molecular taxonomy with cancer stem cell dynamics to predict primary resistance to trastuzumab (Herceptin). Cell Cycle. 2013; 12:225-245.

11. Perou CM, Sørlie T, Eisen MB, van de Rijn M, Jeffrey SS, Rees CA, Pollack JR, Ross DT, Johnsen H, Akslen LA, Fluge O, Pergamenschikov A, Williams C, et al. Molecular portraits of human breast tumours. Nature. 2000; 406:747-752.

12. Sørlie T, Perou CM, Tibshirani R, Aas T, Geisler S, Johnsen H, Hastie T, Eisen MB, van de Rijn M, Jeffrey SS, Thorsen T, Quist H, Matese JC, et al. Gene expression patterns of breast carcinomas distinguish tumor subclasses with clinical implications. Proc Natl Acad Sci U S A. 2001; 98:10869-10874.

13. Sorlie T, Tibshirani R, Parker J, Hastie T, Marron JS, Nobel A, Deng S, Johnsen H, Pesich R, Geisler S, Demeter J, Perou CM, Lønning PE, et al. Repeated observation of breast tumor subtypes in independent gene expression data sets. Proc Natl Acad Sci U S A. 2003; 100:8418-8423.

14. Sotiriou C, Neo SY, McShane LM, Korn EL, Long PM, Jazaeri A, Martiat P, Fox SB, Harris AL, Liu ET. Breast cancer classification and prognosis based on gene expression profiles from a population-based study. Proc Natl Acad Sci U S A. 2003; 100:10393-10398.

15. Bertucci F, Borie N, Ginestier C, Groulet A, CharafeJauffret E, Adélaïde J, Geneix J, Bachelart L, Finetti P, Koki A, Hermitte F, Hassoun J, Debono S, et al. Identification and validation of an ERBB2 gene expression signature in breast cancers. Oncogene. 2004; 23:2564-2575.

16. Laakso M, Tanner M, Nilsson J, Wiklund T, Erikstein B, Kellokumpu-Lehtinen P, Malmström P, Wilking N, Bergh J, Isola J. Basoluminal carcinoma: a new biologically and prognostically distinct entity between basal and luminal breast cancer. Clin Cancer Res. 2006; 12:4185-4191.

17. Liu H, Fan Q, Zhang Z, Li X, Yu H, Meng F. Basal-HER2 phenotype shows poorer survival than basal-like phenotype in hormone receptor-negative invasive breast cancers. Hum Pathol. 2008; 39:167-174.

18. Bhargava R, Beriwal S, Striebel JM, Dabbs DJ. Breast cancer molecular class ERBB2: preponderance of tumors with apocrine differentiation and expression of basal phenotype markers CK5, CK5/6, and EGFR. Appl Immunohistochem Mol Morphol. 2010; 18:113-118.

19. Oliveras-Ferraros C, Vazquez-Martin A, Martin-Castilló B, Pérez-Martínez MC, Cufí S, Del Barco S, Bernado L, Brunet J, López-Bonet E, Menendez JA. Pathway-focused proteomic signatures in HER2-overexpressing breast cancer with a basal-like phenotype: new insights into de novo 
resistance to trastuzumab (Herceptin). Int J Oncol. 2010; 37:669-678.

20. Bagaria SP, Ray PS, Wang J, Kropcho L, Chung A, Sim MS, Shamonki JM, Martino S, Cui X, Giuliano AE. Prognostic value of basal phenotype in HER2-overexpressing breast cancer. Ann Surg Oncol. 2012; 19:935-940.

21. Kaufmann O, Fietze E, Mengs J, Dietel M. Value of p63 and cytokeratin $5 / 6$ as immunohistochemical markers for the differential diagnosis of poorly differentiated and undifferentiated carcinomas. Am J Clin Pathol. 2001; 116:823-830.

22. Krop IE, Burstein HJ. Trastuzumab: qui bono?. J Natl Cancer Inst. 2013; 105:1772-1775.

23. Staaf J, Ringnér M, Vallon-Christersson J, Jönsson G, Bendahl PO, Holm K, Arason A, Gunnarsson H, Hegardt C, Agnarsson BA, Luts L, Grabau D, Fernö M, et al. Identification of subtypes in human epidermal growth factor receptor 2-positive breast cancer reveals a gene signature prognostic of outcome. J Clin Oncol. 2010; 28: 1813-1820.

24. Oliveras-Ferraros C, Corominas-Faja B, Cufí S, VazquezMartin A, Martin-Castillo B, Iglesias JM, López-Bonet E, Martin ÁG Menendez. Epithelial-to-mesenchymal transition (EMT) confers primary resistance to trastuzumab (Herceptin). Cell Cycle. 2012; 11:4020-4032.

25. Oliveras-Ferraros C, Vazquez-Martin A, Cufí S, TorresGarcia VZ, Sauri-Nadal T, Barco SD, Lopez-Bonet E, Brunet J, Martin-Castillo B, Menendez JA. Inhibitor of Apoptosis (IAP) survivin is indispensable for survival of HER2 gene-amplified breast cancer cells with primary resistance to HER1/2-targeted therapies. Biochem Biophys Res Commun. 2011; 407:412-419.

26. Oliveras-Ferraros C, Vazquez-Martin A, Martin-Castillo B, Cufí S, Del Barco S, Lopez-Bonet E, Brunet J, Menendez JA. Dynamic emergence of the mesenchymal CD44(pos) CD24(neg/low) phenotype in HER2-gene amplified breast cancer cells with de novo resistance to trastuzumab (Herceptin). Biochem Biophys Res Commun. 2010; 397:27-33.

27. Cufi S, Corominas-Faja B, Vazquez-Martin A, OliverasFerraros C, Dorca J, Bosch-Barrera J, Martin-Castillo B, Menendez JA. Metformin-induced preferential killing of breast cancer initiating CD44+CD24-/low cells is sufficient to overcome primary resistance to trastuzumab in HER2+ human breast cancer xenografts. Oncotarget. 2012; 3:395-398.

28. Yakirevich E1, Samkari A, Holloway MP, Lu S, Singh K, Yu J, Fenton Altura RA, : Total Survivin and acetylated Survivin correlate with distinct molecular subtypes of breast cancer.Hum Pathol. (MA: 201243865873

29. Honeth G, Bendahl PO, Ringnér M, Saal LH, GruvbergerSaal SK, Lövgren K, Grabau D, Fernö M, Borg A, Hegardt C. The CD44+/CD24- phenotype is enriched in basal-like breast tumors. Breast Cancer Res. 2008; 10:R53.
30. Sarrió D, Rodriguez-Pinilla SM, Hardisson D, Cano A, Moreno-Bueno G, Palacios J. Epithelial-mesenchymal transition in breast cancer relates to the basal-like phenotype. Cancer Res. 2008; 68:989-997.

31. Hennessy BT, Gonzalez-Angulo AM, Stemke-Hale K, Gilcrease MZ, Krishnamurthy S, Lee JS, Fridlyand J, Sahin A, Agarwal R, Joy C, Liu W, Stivers D, Baggerly K, et al. Characterization of a naturally occurring breast cancer subset enriched in epithelial-to-mesenchymal transition and stem cell characteristics. Cancer Res. 2009; 69:4116-4124.

32. Creighton CJ, Chang JC, Rosen JM. Epithelialmesenchymal transition (EMT) in tumor-initiating cells and its clinical implications in breast cancer. J Mammary Gland Biol Neoplasia. 2010; 15:253-260.

33. Taube JH, Herschkowitz JI, Komurov K, Zhou AY, Gupta S, Yang J, Hartwell K, Onder TT, Gupta PB, Evans KW, Hollier BG, Ram PT, Lander ES, et al. Core epithelialto-mesenchymal transition interactome gene-expression signature is associated with claudin-low and metaplastic breast cancer subtypes. Proc Natl Acad Sci U S A. 2010; 107:15449-15454.

34. May CD, Sphyris N, Evans KW, Werden SJ, Guo W, Mani SA. Epithelial-mesenchymal transition and cancer stem cells: a dangerously dynamic duo in breast cancer progression. Breast Cancer Res. 2011; 13:202.

35. Herschkowitz JI, Zhao W, Zhang M, Usary J, Murrow G, Edwards D, Knezevic J, Greene SB, Darr D, Troester MA, Hilsenbeck SG, Medina D, Perou CM, et al. Comparative oncogenomics identifies breast tumors enriched in functional tumor-initiating cells. Proc Natl Acad Sci U S A. 2012; 109:2778-2783.

36. Liu S, Cong Y, Wang D, Sun Y, Deng L, Liu Y, MartinTrevino R, Shang L, McDermott SP, Landis MD, Hong $\mathrm{S}$, Adams A, D'Angelo R, et al. Breast cancer stem cells transition between epithelial and mesenchymal states reflective of their normal counterparts. Stem Cell Reports. 2013; 2:78-91.

37. Casas E, Kim J, Bendesky A, Ohno-Machado L, Wolfe CJ, Yang J. Snail2 is an essential mediator of Twist1-induced epithelial mesenchymal transition and metastasis. Cancer Res. 2011; 71:245-254.

38. Gyorffy B, Lanczky A, Eklund AC, Denkert C, Budczies J, Li Q, Szallasi Z. An online survival analysis tool to rapidly assess the effect of 22,277 genes on breast cancer prognosis using microarray data of 1809 patients, Breast Cancer Res Treatment. 2010; 123:725-731.

39. Gyorffy B, Lanczky A, Szallasi Z. Implementing an online tool for genome-wide validation of survival-associated biomarkers in ovarian-cancer using microarray data of 1287 patients, Endocrine-Related Cancer. 2012; 19:197-208. 